\title{
TMEM119 silencing inhibits cell viability and causes the apoptosis of gastric cancer SGC-7901 cells
}

\author{
PEIFEN ZHENG, WEIFENG WANG, MUXI JI, QIN ZHU, YULIANG FENG, \\ FENG ZHOU and QIAONA HE \\ Department of Gastroenterology, Zhejiang Hospital, Hangzhou, Zhejiang 310013, P.R. China
}

Received September 26, 2017; Accepted February 2, 2018

DOI: $10.3892 / 01.2018 .8358$

\begin{abstract}
Gastric cancer is the second major cause of death associated with cancer and ranks among the top four cancers diagnosed worldwide. Previous findings identified the association of transmembrane proteins (TMEMs) with tumorigenesis of various types of cancer, including breast, liver and kidney cancer. However, the expression and the biological function of TMEMs, especially TMEM119, and its possible molecular mechanism in gastric cancer remain less understood. CCK-8 and flow cytometric analysis was employed to examine the viability and apoptosis of gastric adenocarcinoma SGC-7901 and AGS cells, gastric carcinoma MKN45 cells, as well as gastric epithelial cell lines GES-1 after transfection with TMEM119-siRNA (siTMEM119), respectively. Quantitative PCR, western blot analysis and immunohistochemistry was performed to detect the expression levels of TMEM119, Bax, Bcl-2 and caspase-3. The results showed that, TMEM119 was elevated with the highest expression detected in SGC-7901 cells compared to AGS cells, MKN45 cells, as well as GES-1. TMEM119 silencing in the gastric cancer cell line, SGC-7901, significantly inhibited cell viability and induced apoptosis. The downregulation of TMEM119 exhibited reduced levels of Bcl-2 and higher levels of Bax and caspase-3 in SGC-7901 cells. These results suggest that TMEM119 is useful in the treatment of gastric cancer.
\end{abstract}

\section{Introduction}

Gastric cancer is a well-known malignant tumor, which is harmful to human health. The incidence of gastric cancer increases significantly with age, and the peak age of onset is 50-80 years, although it is showing a younger tendency every year (1). The proportion of 19- to 35-year-old patients

Correspondence to: Dr Peifen Zheng, Department of Gastroenterology, Zhejiang Hospital, 12 Lingyin Road, Hangzhou, Zhejiang 310013, P.R. China

E-mail: 17706413537@163.com

Key words: transmembrane protein 119, gastric cancer, apoptosis, $\mathrm{Bax} / \mathrm{Bcl}-2$, caspase-3 with gastric cancer has increased from 1.7 to $3.3 \%$ in the past 40 years (1). Although surgical resection and adjuvant chemotherapy and radiotherapy are primarily employed in the treatment of the disease, most patients with gastric cancer succumb to tumor recurrence and metastasis (2-4), and the five-year survival rate is $<24 \%$ (5). The occurrence of gastric cancer is influenced by many factors $(6,7)$. The specific molecular mechanism of the occurrence of gastric cancer remains to be determined. Current use of traditional cytotoxic chemotherapy agents for gastric cancer is limited (8). At present, many studies involving molecular pathways aim to identify new targets to treat gastric cancer. The development of molecular diagnostic science has further supported the finding of new molecular targets.

Transmembrane protein 119 (TMEM119) belongs to the transmembrane proteins (TMEMs) family. Differential regulation of TMEMs is observed in many types of cancer. TMEM176A and TMEM176B were significantly elevated in lymphoma and associated with certain cancer pathology (9). TMEM72 and TMEM116 were downregulated in metastatic clear cell renal cell carcinoma (ccRCC) tissue, and TMEM30B and TMEM45B were downregulated in advanced-stage samples of ccRCC, suggesting that TMEM could be utilized as a potential predictor for metastases and cancer progression (10). TMEM45B was overexpressed in lung cancer and the knockdown of TMEM45B suppressed lung cancer cell invasion, migration and proliferation and caused cell cycle arrest and cell apoptosis (11). TMEM106A is often methylated in human gastric cancer and TMEM106A upregulation suppressed cell growth and induced apoptosis in gastric cancer cell lines (12). TMEM119 is important in bone formation and normal bone mineralization. Recently, TMEM119 induced the transshipment and differentiation of muscle cells into osteoblasts by enhancing the interaction of BMP2 and the bmp-runx2 pathway (13). TMEM119 was increased in osteosarcoma, connected with tumor size, clinical stage and overall survival time, and associated with cell cycle, metastasis, apoptosis as well as TGF- $\beta$ signaling in osteosarcoma cell lines (14). It was found that human microglia, along with neuroblastoma cells and Alzheimer's disease, expressed high levels of TMEM119 mRNA (15). However, the functions of TMEM119 in gastric cancer remain to be investigated.

In the present study, the impact of TMEM119 on cell viability and apoptosis of SGC-7901 and AGS cells, gastric 
carcinoma MKN45 cells, as well as gastric epithelial cell lines GES-1 was investigated. It was found that there was more TMEM119 in gastric cancer tissues than in normal tissues and knockdown of TMEM119 significantly curbed viability and induced cell apoptosis of SGC-7901, AGS cells and MKN45 cells. In addition, TMEM119 downregulation decreased the expression of $\mathrm{Bcl}-2$ but increased the expression of Bax and caspase-3. Our results demonstrate a significant role of TMEM119 in the regulation of SGC-7901 cell viability and apoptosis.

\section{Materials and methods}

Analysis of bioinformatics. Data regarding gene expression were obtained from The Cancer Genome Atlas website (TCGA; https://cancergenome.nih.gov/).

Patient specimens. Tumor and general gastric specimens were obtained from 90 gastric cancer patients who experienced surgery at the Zhejiang Hospital (Hangzhou, China) from June, 2010 to April, 2015. The research program was approved by the Zhejiang Hospital Ethics Committee. All the participants in this study provided informed consent.

Cell culture. Gastric adenocarcinoma SGC-7901 and AGS cells, gastric carcinoma MKN45 cells, as well as gastric epithelial cell lines GES-1 (The Cell Bank of Type Culture Collection of Chinese Academy of Sciences, Shanghai, China) were used in the present study. The cell lines were cultured in DMEM medium followed by $10 \%$ (v/v) fetal bovine serum (Life Technologies; Thermo Fisher Scientific, Inc., Waltham, MA, USA) at $37^{\circ} \mathrm{C}$ and $5 \%$ of the $\mathrm{CO}_{2}$ in an incubator.

RNA interference. TMEM119 mRNA (5'-ugggauaguggacuuc uuc-3') and non-specific interference siRNA sequences (control siRNA; 5'-uucuccgaacgucacgu-3') siRNA were synthesized and transfected with Lipofectamine 2000 (Invitrogen; Thermo Fisher Scientific, Inc.) as per the manufacturer's protocol. Assays were performed $48 \mathrm{~h}$ after transfection. The TMEM119 mRNA expression in 249 gastric cancer tissues and 33 normal gastric tissues from the TCGA dataset was first examined.

Cell Counting kit-8 (CCK-8) assay. Cell viability was determined using a Cell Counting kit-8 (Dojindo Molecular Technologies, Inc., Kumamoto, Japan), where the substrate was 2-(2-methoxy-4-nitrophenyl)-3-(4-nitrophenyl)-5-(2, 4-disulfophenyl)-2H-tetrazolium monosodium salt (WST-8). After the cells were incubated for 12, 24, 48 and 72 h, CCK-8 solution $(10 \mu \mathrm{l})$ was placed into each well of the plate. The plates were incubated at $37^{\circ} \mathrm{C}, 5 \% \mathrm{CO}_{2}$, for $4 \mathrm{~h}$.

Cell apoptosis assay. In accordance with the manufacturer's protocol, SGC-7901 cell apoptosis was analyzed using the Annexin V-FITC/iodide (PI) cell apoptosis kit (BD Biosciences, Franklin Lakes, NJ, USA). The cells were washed with phosphate buffered salline (PBS) three times, followed by trypsin digestion, centrifugation at $400 \mathrm{x} \mathrm{g}$ at room temperature for $10 \mathrm{~min}$, and were adjusted to $5 \times 10^{4} / \mathrm{ml}$, after which they were suspended in binding buffer containing Annexin V-FITC and PI. The fluorescence intensity was measured via flow cytometry (BD Biosciences) after $10 \mathrm{~min}$ of incubation in the dark.

Reverse transcription and quantitative PCR. Using TRIzol reagent (Invitrogen; Thermo Fisher Scientific, Inc.) RNA was extracted from patient tissue samples and cell lines, and were treated with DNase I (Roche Diagnostics, Indianapolis, IN, USA) to eliminate residual DNA as per the manufacturer's protocol. The Prime Script PTMP RT reagent kit (Perfect Real Time; Takara Biotechnology Co., Ltd., Dalian, China) was used to generate cDNA, and reverse transcribed with $1 \mu \mathrm{g}$ total RNA, followed by SYBR-Green qPCR Master (Thermo Fisher Scientific, Inc.) to determine quantitative PCR in accordance with the manufacturer's protocol. Primers used to amplify the coding region were: 5'-CTGGCCTTTCTGCTGATGTTC-3' (forward) and 5'-TCACTCTGGTCCACGTACTTC-3' (reverse), for TMEM119, and 5'-CACCCACTCCTCCACCTT TG-3' (forward) and 5'-CCACCACCCTGTTGCTGTAG-3' (reverse) for GAPDH. GAPDH mRNA levels were employed for normalization while the $2^{-\Delta \Delta \mathrm{Cq}}$ method was employed to calculate the expression changes.

Western blot analysis. To determine the protein expression level, complete cell extracts were prepared in the lysis buffer. After culture, the medium was removed, SGC-7901 cells were obtained and washed with PBS, and were immediately lysed in a buffer containing $20 \mathrm{mM}$ Tris- $\mathrm{HCl}$. The total protein was quantitatively determined using BCA protein. Loading buffer was added into the cytoplasm extract and boiled for approximately $5 \mathrm{~min}$. The gel was then separated from each sample of the same amount by $10 \%$ SDS-PAGE, and transferred to the nylon membrane. Following incubation for $1.5 \mathrm{~h}$ with a $5 \%$ degreasing emulsion in the fresh block buffer, rabbit anti-human Bax, Bcl-2, caspase-3 and GAPDH polyclonal antibodies (1:500; cat. nos. PA5-11378, PA5-20068, PA5-16332, and PA1-987-HRP; Thermo Fisher Scientific, Inc.) and rabbit anti-human TMEM119 polyclonal antibody (1:500; cat. no. ab185333; Abcam,Cambridge, MA, USA) were incubated overnight at $4^{\circ} \mathrm{C}$ in freshly prepared TBST containing 5\% skim milk. The membrane was cleaned 3 times with the TBST and then incubated with the secondary antibody at room temperature for $2 \mathrm{~h}$. Specific proteins were tested with enhanced chemiluminescence after goat anti-rabbit polyclonal secondary antibody (1:1,000; cat. no. 31460; Thermo Fisher Scientific, Inc.) binding.

Immunohistochemistry. Tissue sections $(5 \mu \mathrm{m})$ were cut and mounted on slides. After dewaxing and rehydration, the sections were antigen-retrieved in $10 \mathrm{~mm}$ citrate buffer for $5 \mathrm{~min}$ at $100^{\circ} \mathrm{C}$. Endogenous peroxidase activity and non-specific antigens were blocked with $3 \%$ hydrogen peroxide and serum, followed by incubation with rabbit anti-human TMEM119 polyclonal antibody (1:500; cat. no. HPA051870; Sigma-Aldrich; Merck KGaA, Darmstadt, Germany) over-night at $4^{\circ} \mathrm{C}$. The slides were then incubated with goat anti-rabbit secondary polyclonal antibody (1:2,000; cat. no. A0545; Sigma-Aldrich; Merck KGaA), developed using 3,3'-diaminobenzidine solution and counterstained with hematoxylin.

Statistical analysis. Statistical analysis was performed using the ANOVA and post-hoc test was SNK test. $\mathrm{P}<0.05$ was 
A

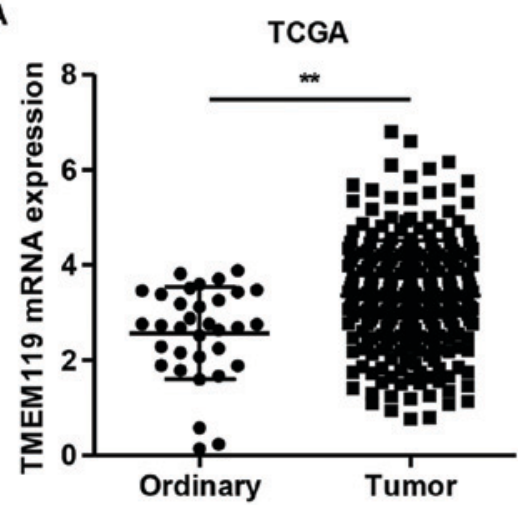

B

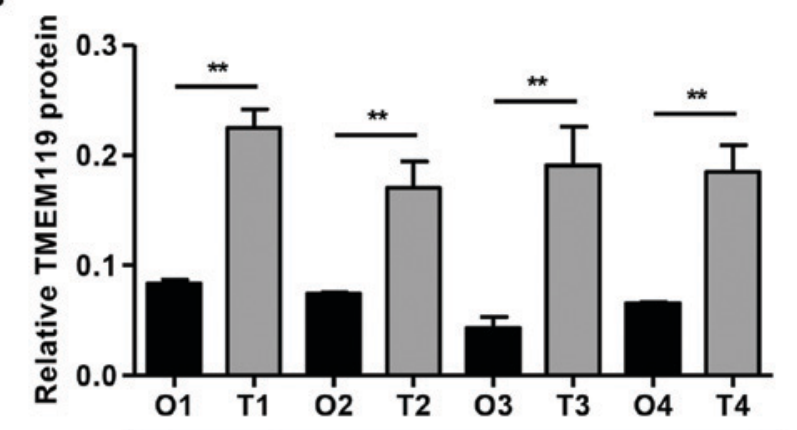

TMEM119

GAPDH

C

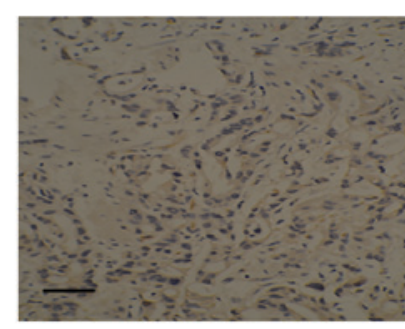

Ordinary

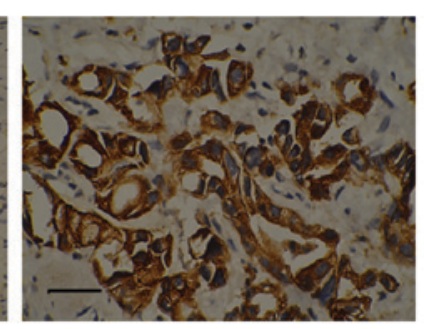

Tumor
D

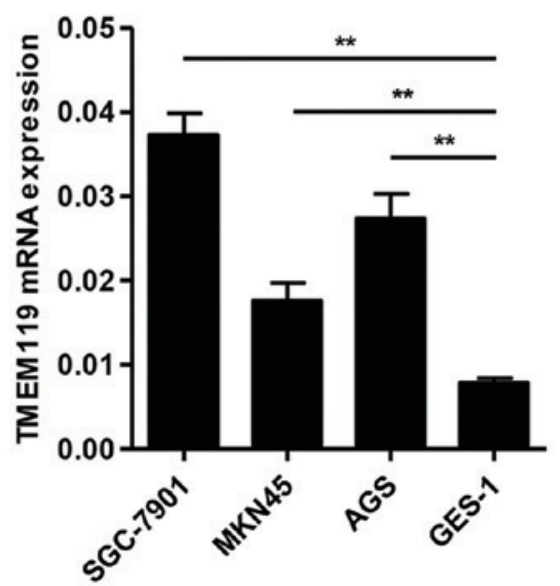

Figure 1. Expression of TMEM119 in gastric cancer tissues. (A) An increase of TMEM119 mRNA expression in 249 gastric cancer tissues compared with 33 ordinary gastric tissues from the TCGA database. (B) TMEM119 protein expression was increased in four gastric cancer tissues as compared to the corresponding normal gastric tissues, as indicated in the western blot analysis. (C) Immunohistochemical analysis reveals tumor upregulation (magnification, $\mathrm{x} 400$ ). (D) TMEM119 mRNA expression was higher in all the gastric cancer cell lines compared to GES-1 cells, with the highest expression detected in SGC-7901 cells. Low power (x200). Scale bars, $100 \mu \mathrm{m} .{ }^{* *} \mathrm{P}<0.01$. TMEM119, transmembrane protein 119; TCGA, The Cancer Genome Atlas.

considered to indicate a statistically significant analysis. All statistical analyses were carried out with the GraphPad Prism software (GraphPad Software, Inc., La Jolla, CA, USA).

\section{Results}

TMEM119 is upregulated in gastric cancer tissues and cell lines. The TMEM119 mRNA expression in 249 gastric cancer tissues and 33 normal gastric tissues from the TCGA dataset was first examined (Fig. 1A). TMEM119 showed a significantly higher expression in gastric cancer tissues than in ordinary tissues. TMEM119 protein levels in gastric cancer tissues and normal gastric tissues obtained from the Zhejiang Hospital were evaluated using western blot analysis. TMEM119 protein expression was increased in all four gastric cancer tissues compared with the normal gastric tissues (Fig. 1B). Immunohistochemical analysis revealed that the expression of TMEM119 was significantly upregulated in gastric cancer tissues compared with the corresponding normal gastric tissues (Fig. 1C). Quantitative PCR analysis showed a higher expression of TMEM119 in all the gastric cancer cell lines compared to GES-1 cells, with the highest expression detected in SGC-7901 cells. The SGC-7901 cells were therefore used for subsequent experiments (Fig. 1D). These data further indicate that TMEM119 was significantly upregulated in gastric cancer, and that TMEM119 may facilitate gastric cancer carcinogenesis.

TMEM119 silencing inhibits SGC-7901 cell viability. To validate the role of TMEM119 in gastric cancer in vitro, a special siRNA targeting TMEM119 and a scramble siRNA (control siRNA) were transfected into the gastric cancer cell lines, SGC-7901. According to Fig. 2A-C, siTMEM119 transfection significantly decreased the mRNA and protein expression of TMEM119 by 79.8 and $73.1 \%$ in SGC-7901, respectively. Moreover, the cell viability after siTMEM119 transfection was also measured by CCK-8 assay. Our results showed that siTMEM119 transfection significantly inhibited SGC-7901 cell viability by $15.8,20.6,31.9$ and $48.6 \%$ at 12,24 , 48 and 72 h, respectively (Fig. 2D).

TMEM119 silencing induces SGC-7901 cell apoptosis. To determine the function of TMEM119 in the cell apoptosis of SGC-7901, a flow cytometry assay was performed. As shown in Fig. 3A and B, siTMEM119 transfection significantly increased apoptotic cells of SGC-7901 by 10.2-fold. Additionally, some proteins associated with apoptosis, including Bax, Bcl-2 and caspase-3, were also measured by western blot analysis. We found that siTMEM119 transfection greatly decreased Bcl-2 expression but increased the expression of Bax and caspase- 3 
A

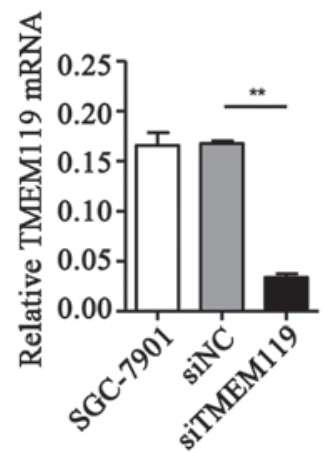

B

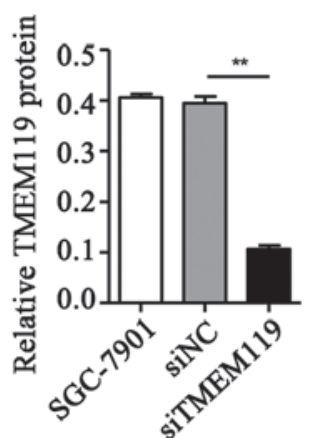

C

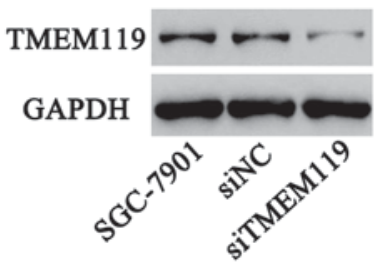

D

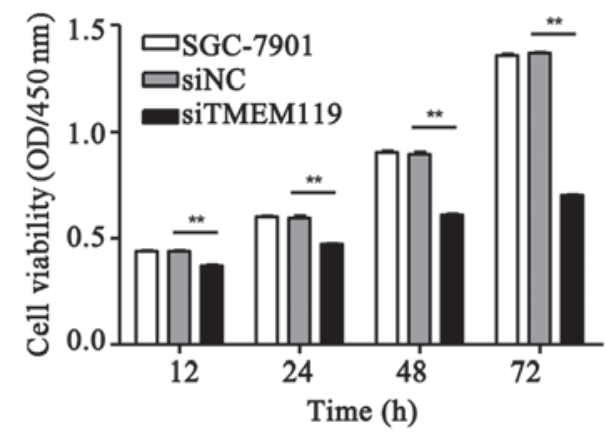

Figure 2. TMEM119 silencing inhibited SGC-7901 cell viability. After transfection of SGC-7901 cells with TMEM119-siRNA (siTMEM119), the expression of TMEM119 was measured by (A) quantitative PCR and western blot analysis. (B and C) Cell viability was measured using the CCK-8 assay. (D) ** P $<0.01$, compared to SGC-7901. TMEM119, transmembrane protein 119; PCR, polymerase chain reaction; CCK-8, Cell Counting Kit-8.

A

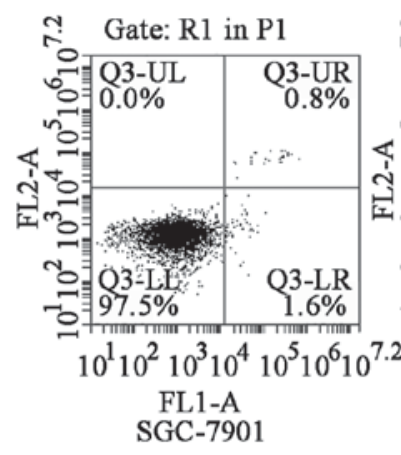

C

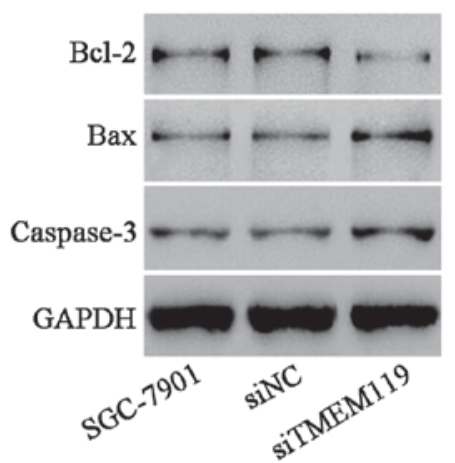

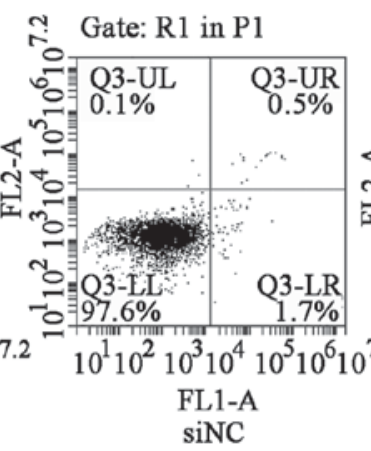

D

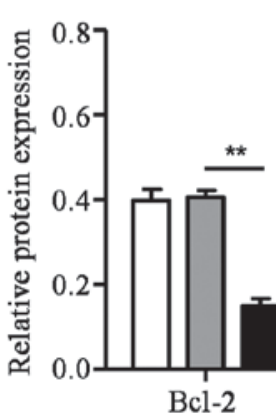

B
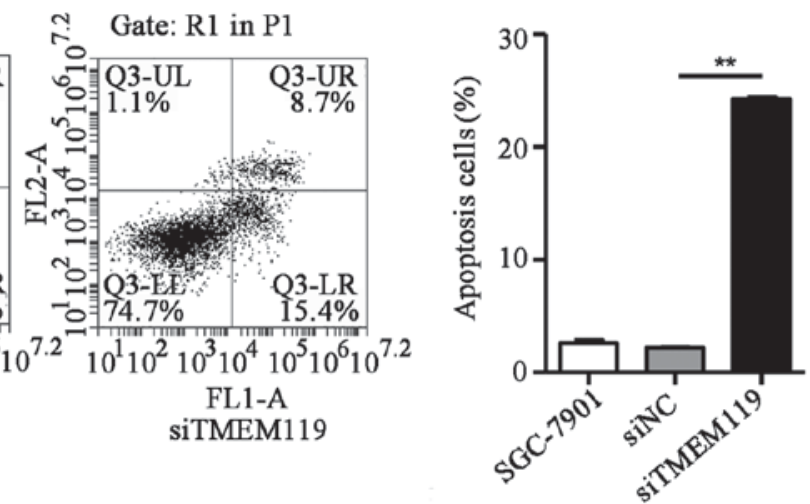

Figure 3. TMEM119 silencing induced SGC-7901 cell apoptosis. After transfection of SGC-7901 cells with TMEM119-siRNA (siTMEM119), cell apoptosis was measured by (A and B) flow cytometry assay and the expression of Bcl-2, Bax as well as caspase-3 was measured by (C and D) western blot analysis. ${ }^{* * *} \mathrm{P}<0.01$, compared to SGC-7901. TMEM119, transmembrane protein 119.

in SGC-7901 cells (Fig. 3C and D). According to the results, TMEM119 silencing induces SGC-7901 cell apoptosis through an increase of Bax/Bcl-2 ratio and caspase-3 expression.

\section{Discussion}

TMEM proteins have been recently extensively studied in different types of malignant tumors (9-11) and other members of TMEM proteins have been found to be overexpressed in gastric cancer. TMEM106A is often methylated in human gastric cancer and TMEM106A upregulation suppressed cell growth and induced apoptosis in gastric cancer cell lines (12). TMEM16A overexpression contributes to tumor invasion and poor prognosis of human gastric cancer (16). However, knowledge concerning the aberrant expression and potential role of TMEM119 in gastric cancer is lacking. In the present study, we demonstrated that TMEM119 was overexpressed in gastric cancer tissues by analyzing independent dataset downloaded from the TCGA website, and our western blot analysis results on 90 pairs of gastric cancer as well as normal 
tissues. TMEM119 silencing inhibited cell viability and caused cell apoptosis of gastric cancer cells.

Previous findings revealed that TMEM119 was overexpressed in osteosarcoma tissues and the knockdown of TMEM119 suppressed the migratory and invasive abilities of osteosarcoma cells and osteosarcoma cell growth in vitro and in vivo, causing G0/G1-phase arrest and apoptosis (14). In line with the previous study, it was identified that TMEM119 was significantly upregulated in gastric cancer tissues compared to ordinary tissues from the TCGA database and our hospital samples. TMEM119 silencing significantly prevented SGC-7901 cell viability as well as induced cell apoptosis. There are several pathways to induce cancer cell apoptosis, such as regulating apoptosis-related genes, and changing intracellular $\mathrm{Ca}^{2+}$ concentration in endoplasmic reticulum and mitochondrial pathway $(17,18)$. Apoptosis is a complex signaling pathway in which there are a number of genes responsible for pro- and anti-apoptosis, including the Bax, Fas, p38, p53 and caspase family and the Bcl-2 family $(19,20)$. Numerous studies have shown that Bcl-2 plays a key role in the complex cell death signaling pathways $(21,22)$. Bcl-2 family proteins contain anti- as well as pro-apoptotic genes. $\mathrm{Bcl}-2$ belongs to anti-apoptotic genes, while Bax belongs to pro-apoptotic genes, and a ratio between them determines cell apoptosis or not. The anti-apoptotic role of Bcl-2 is very significant in cell life and cell response to adverse stimulation (23). In the present study, our results revealed that TMEM119 silencing significantly caused apoptosis of SGC-7901 cells and an increase of the Bax/Bcl-2 ratio as well as caspase-3 expression.

In conclusion, we have demonstrated that TMEM119 was increased in gastric cancer tissues and is essential in regulating cell viability and apoptosis in vitro. In addition, the molecular mechanism involved may be the increase in the Bax/Bcl-2 ratio as well as caspase-3 expression, indicating novel targets for therapeutic interventions to prevent gastric cancer.

\section{Acknowledgements}

Not applicable.

\section{Funding}

The present study was funded by the Natural Science Foundation of Zhejiang Province (Y17H030031) and the Medical and Health Science and Technology Plan of Zhejiang Province (2016ZDA001).

\section{Availability of data and materials}

The datasets used and/or analyzed during the current study are available from the corresponding author on reasonable request.

\section{Authors' contributions}

PZ and WW wrote the the manuscript and made contributions to cell culture. MJ and QZ performed and analysed quantitative PCR. YF helped with western blot analysis. FZ contributed in immunohistochemistry. QH conducted cell apoptosis assay. All authors read and approved the final manuscript.

\section{Ethics approval and consent to participate}

The research program was approved by the Zhejiang Hospital Ethics Committee (Hangzhou, China). All the participants in this study provided informed consent.

\section{Consent for publication}

Not applicable.

\section{Competing interests}

The authors declare that they have no competing interests.

\section{References}

1. Ferlay J, Shin HR, Bray F, Forman D, Mathers C and Parkin DM: Estimates of worldwide burden of cancer in 2008: GLOBOCAN 2008. Int J Cancer 127: 2893-2917, 2010.

2. Wei J, Wu ND and Liu BR: Regional but fatal: Intraperitoneal metastasis in gastric cancer. World J Gastroenterol 22: 7478-7485, 2016.

3. Jeon MY, Park JC, Hahn KY, Shin SK, Lee SK and Lee YC: Long-term outcomes after noncurative endoscopic resection of early gastric cancer: The optimal time for additional endoscopic treatment. Gastrointest Endosc: Oct 12, 2017 (Epub ahead of print).

4. Peixoto A, Santos-Antunes J, Silva M and Macedo G: Duodenal stump recurrence of gastric adenocarcinoma after subtotal gastrectomy. Rev Esp Enferm Dig 108: 739-740, 2016.

5. Yasumoto K, Koizumi K, Kawashima A, Saitoh Y, Arita Y, Shinohara K, Minami T, Nakayama T, Sakurai H, Takahashi Y, et al: Role of the CXCL12/CXCR4 axis in peritoneal carcinomatosis of gastric cancer. Cancer Res 66: 2181-2187, 2006.

6. Ajani JA: Evolving chemotherapy for advanced gastric cancer. Oncologist 10: 49-58, 2005.

7. Karimi P, Islami F, Anandasabapathy S, Freedman ND and Kamangar F: Gastric cancer: Descriptive epidemiology, risk factors, screening, and prevention. Cancer Epidemiol Biomarkers Prev 23: 700-713, 2014.

8. Charalampakis N, Elimova E, Shimodaira Y, Shiozaki H, Wadhwa R and Ajani JA: Biologics in combination with chemotherapy for gastric cancer: Is this the answer? Expert Opin Pharmacother 16: 955-960, 2015.

9. Cuajungco MP, Podevin W, Valluri VK, Bui Q, Nguyen VH and Taylor K: Abnormal accumulation of human transmembrane (TMEM)-176A and 176B proteins is associated with cancer pathology. Acta Histochem 114: 705-712, 2012.

10. Wrzesiński T, Szelag M, Cieślikowski WA, Ida A, Giles R, Zodro E, Szumska J, Poźniak J, Kwias Z, Bluyssen HA, et al: Expression of pre-selected TMEMs with predicted ER localization as potential classifiers of ccRCC tumors. BMC Cancer 15: 518, 2015.

11. Hu R, Hu F, Xie X, Wang L, Li G, Qiao T, Wang M and Xiao H: TMEM45B, up-regulated in human lung cancer, enhances tumorigenicity of lung cancer cells. Tumour Biol 37: 12181-12191, 2016.

12. Xu D, Qu L, Hu J, Li G, Lv P, Ma D, Guo M and Chen Y: Transmembrane protein 106A is silenced by promoter region hypermethylation and suppresses gastric cancer growth by inducing apoptosis. J Cell Mol Med 18: 1655-1666, 2014.

13. Hisa I, Inoue Y, Hendy GN, Canaff L, Kitazawa R, Kitazawa S, Komori T, Sugimoto T, Seino S and Kaji H: Parathyroid hormone-responsive Smad3-related factor, Tmem119, promotes osteoblast differentiation and interacts with the bone morphogenetic protein-Runx2 pathway. J Biol Chem 286: 9787-9796, 2011.

14. Jiang ZH, Peng J, Yang HL, Fu XL, Wang JZ, Liu L, Jiang JN, Tan YF and Ge ZJ: Upregulation and biological function of transmembrane protein 119 in osteosarcoma. Exp Mol Med 49: 329, 2017.

15. Satoh J, Kino Y, Asahina N, Takitani M, Miyoshi J, Ishida T and Saito Y: TMEM119 marks a subset of microglia in the human brain. Neuropathology 36: 39-49, 2016. 
16. Liu F, Cao QH, Lu DJ, Luo B, Lu XF, Luo RC and Wang XG: TMEM16A overexpression contributes to tumor invasion and poor prognosis of human gastric cancer through TGF- $\beta$ signaling. Oncotarget 6: 11585-11599, 2015.

17. Kim KY, Cho HJ, Yu SN, Kim SH, Yu HS, Park YM, Mirkheshti N, Kim SY, Song CS, Chatterjee B, et al: Interplay of reactive oxygen species, intracellular $\mathrm{Ca} 2+$ and mitochondrial homeostasis in the apoptosis of prostate cancer cells by deoxypodophyllotoxin. J Cell Biochem 114: 1124-1134, 2013.

18. Sharifi S, Barar J, Hejazi MS and Samadi N: Doxorubicin Changes Bax /Bcl-xL Ratio, Caspase- 8 and 9 in breast cancer cells. Adv Pharm Bull 5: 351-359, 2015.

19. Song Y, Li X, Li Y, Li N, Shi X, Ding H, Zhang Y, Li X, Liu $G$ and Wang Z: Non-esterified fatty acids activate the ROS-p38-p53/Nrf2 signaling pathway to induce bovine hepatocyte apoptosis in vitro. Apoptosis: 19: 984-997, 2014.

20. Gao J, Gao J, Qian L, Wang X, Wu M, Zhang Y, Ye H, Zhu S, Yu Y and Han W: Activation of p38-MAPK by CXCL4/CXCR3 axis contributes to p53-dependent intestinal apoptosis initiated by 5-fluorouracil. Cancer Biol Ther 15: 982-991, 2014.
21. Czabotar PE, Lessene G, Strasser A and Adams JM: Control of apoptosis by the BCL-2 protein family: Implications for physiology and therapy. Nat Rev Mol Cell Biol 15: 49-63, 2014.

22. Akl H, Vervloessem T, Kiviluoto S, Bittremieux M, Parys JB, De Smedt $\mathrm{H}$ and Bultynck G: A dual role for the anti-apoptotic Bcl-2 protein in cancer: Mitochondria versus endoplasmic reticulum. Biochim Biophys Acta 1843: 2240-2252, 2014.

23. Crew KD and Neugut AI: Epidemiology of gastric cancer. World J Gastroenterol 12: 354-362, 2006.

(i) $\Theta$ This work is licensed under a Creative Commons Attribution-NonCommercial-NoDerivatives 4.0 International (CC BY-NC-ND 4.0) License. 